МАТРАЕВА Л.В., АЛЯБЬЕВА А.В.

МАКРОЭКОНОМИЧЕСКИЕ ЭФФЕКТЫ ПРЯМОГО ИНОСТРАННОГО ИНВЕСТИРОВАНИЯ В РЕГИОНЫ РОССИЙСКОЙ ФЕДЕРАЦИИ

УДК 339.72+330.88

МАТРАЕВА ЛИЛИЯ ВАЛЕРИЕВНА

д.э.н., профессор кафедры «Экономическая теория и мировая экономика» ФФГБОУ ВО

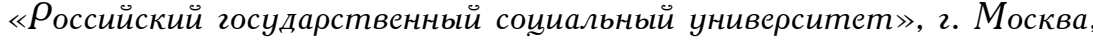
e-mail: matraeva@rambler.ru

АИЯБЬЕВА АНАСТАСИЯ ВИКТОРОВНА

магисто кафедры «Экономическая теория и мировая экономика» ФГБОУ ВО «оссийский государственный социальный университет», г. Москва, e-mail: nastya-alyabeva@mail.ru

\title{
МАКРОЭКОНОМИЧЕСКИЕ ЭФФЕКТЫ ПРЯМОГО ИНОСТРАННОГО ИНВЕСТИРОВАНИЯ В РОССИЙСКУЮ ФЕАЕРАЦИЮ
}

\begin{abstract}
Аннотауия. Џель работы. Џелью работы является исследование макроэкономических эффектов прямого иностранного инвестирования в регионы РФФ. Метод или методология проведения работы. Исследование основывается на общенаучной методологии, которая предусматривает применение системного подхода к решению проблем. Основой данной работы являются фундаментальные труды отечественных и зарубежных ученых по

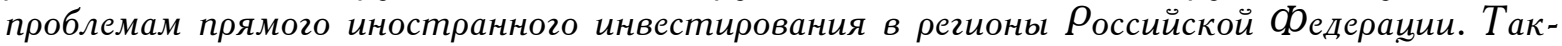
же в работе используются спещиальные экономико-статистические методы моделирования макроэкономических процессов, такие как модель мультипликатора-акселератора и модель оценки эффекта мультипликации иностранных инвестищий посредством коэффиииентов эластичности. Результаты. С 2003 по 2016 год в отдача иностранных инвестиций была выше национальных в несколько раз, однако ино-

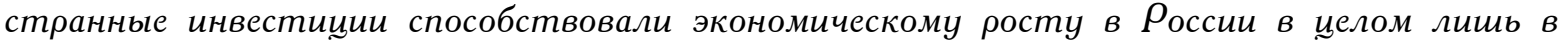
период с 1999 по 2003 год. Выявленное противоречие объясняется сложившейся дифференциачией потоков и различным влиянием прямых иностранных инвестииий на экономический рост различных регионов $О Ф D$. Область применения результатов. Полученные результаты исследования способствуют повышению объективности принятия управленческих решений в отношении стимулирования прямых иностранных инвестищий на общегосударственном уровне с учетом фазы жизненного иикла инвестиционного процесса и характера проявления описанных эффектов от ПИИ на территории РФD. Выводы. В сложившейся ситуащии, на наш взгляд, более эффективной становится модель адресной региональной государственной политики стимулирования иностранных инвестищий, которая позволит максимально использовать положительный эффект в конкретных регионах $Р Ф$ с учетом стадии жизненного цикла иностранных инвестииий в рамках данной территории и нивелировать отрищательные эффекты, наблюдающиеся в других региоHax.
\end{abstract}

Ключевые слова: иностранные инвестиции, эффекты иностранных инвестииий, экономический рост.

MATRAEVA LILIYA VALERIEVNA

Doctor of Economic Sciences, $\rho_{\text {rofessor of the Department of "Economic Theory and International }}$ Economics" of FSBEI of HE "Russian State Social University" in Moscow, e-mail:matraeva@rambler.ru

AL YABIEVA ANASTASIA VICTOROVNA

Master's Degree Student of the Department of "Economic Theory and International Economics" of FSBEI of HE "Russian State Social University", Moscow, e-mail: nastya-alyabeva@mail.ru

\section{MACROECONOMIC EFFECTS OF DIRECT FOREIGN INVESTING IN THE RUSSIAN FEDERATION}


Abstract. The goal of the study. The goal of the study is researching macroeconomic effects of direct foreign investment into the regions of the Russian Federation. The method or methodology of completing the study. The study is based on the general scientific method that stipulates the use of a systemic approach to solving problems. The foundation of this study are fundamental works of domestic and foreign scientists on the issues of direct foreign investment into the regions of the Russian Federation. Also, in this study we use special economic-statistical methods of modeling of macroeconomic processes such as a model of a multiplier-accelerator and a model of evaluation of the multiplying effect of foreign investments through an index of elasticity. The results. From 2003 to 2016 in the Russian Federation the incremental return on capital for foreign investments was higher than that of the national one by several fold, however foreign investments stipulated the economic growth in Russia overall only for the period between 1999 and 2003. The contradiction found is explained by the established differentiation of streams and different impacts of direct foreign investments on the economic growth of different regions of the Russian Federation. The area of application of the results. The results of the study obtained stipulate the improvement of objectiveness of making managerial decisions towards stimulating direct foreign investments on the overall state level taking into account the phase of the lifecycle of the investment process and the character of manifestation of the described effects from FDI on the territory of the Russian Federation. The conclusions. In the situation that got established, as per our opinion, the model of a targeted regional state policy of promoting foreign investments becomes more effective. It will allow to use the positive effect in specific regions of the Russian Federation to the maximum, taking into account the stage of the life cycle of foreign investments in the context of this territory and offset the negative effects that are observed in other regions.

Keywords: Foreign Investments, Effects of Foreign investments, Economic growth.

В современном мире интенсивно идут процессы глобализации и интеграции экономических связей, увеличивается мобильность капитала. Достаточно продолжительное время в экономической науке доминировали подходы, основанные только на положительных эффектах прямого иностранного инвестирования, но существует и диаметрально противоположная точка зрения, предполагающая совместное наличие положительных и отрицательных эффектов или вовсе отсутствие благоприятного воздействия для страны-реципиента. В данной работе автором на основе статистического анализа и макроэкономического моделирования исследуются эффекты прямых иностранных инвестиций в экономику Российской Федерации. Была изучена гипотеза о том, что иностранные инвестиции способствуют экономическому росту в развивающихся странах. Эта гипотеза была проверена на основе тестирования двух гипотез. Первая связана с оценкой экономических последствий на страну-донора на основе модифицированной разностной модели мультипликатора-акселератора. Вторая гипотеза основана на проверке влияния иностранных инвестиций на экономику принимающей страны на основе коэффициента эластичности внешних и внутренних потоков инвестиций. В исследовании используются годовые данные о потоках прямых иностранных инвестиций в Российской Федерации в период с 1999 по 2015 год. В рамках исследования выделяются несколько целевых групп для анализа: регионы, являющиеся активными донорами инвестиций, и регионы, в которых потоки иностранных инвестиций незначительны. Информационной основой исследования являются опубликованные официальные отчеты, доклады и статистические базы международных организаций, таких как ЮНКТАД, Группа Всемирного банка, базы данных Росстата. Сравнение эффектов от иностранных инвестиций в данных целевых группах позволило автору дать более точную характеристику гипотез о характере последствий иностранного инвестирования в регионы Российской Федерации.

1. Систематизация научных взглядов относительно воздействия прямых иностранных инвестиций на экономику принимающей страны

Несмотря на то, что вопросы иностранного инвестирования не являются особенно новыми для экономической науки, к ним достаточно пристально обращено научное внимание с начала 70-х прошлого века до настоящего времени. Это в первую очередь противоречит суждениям об эффектах прямого иностранного инвестирования (далее - ПИИ), присутствующих в научной литературе. Некоторые ученые считают полезным последствия ПИИ в отношении экономического развития, в то время как другие утверждают, что ПИИ препятствуют ему (табл. 1). 
МатРАЕВА Л.В., АЛЯБЬЕВА А.В.

МАКРОЭКОНОМИЧЕСКИЕ ЭФФЕКТЫ ПРЯМОГО ИНОСТРАННОГО ИНВЕСТИРОВАНИЯ В РЕГИОНЫ РОССИЙСКОЙ ФЕДЕРАЦИИ

Систематизация теоретических подходов анализа воздействия ПИИ на экономику страны-реципиента

\begin{tabular}{|c|c|}
\hline Автор & Сущность теории \\
\hline Т. Озава $[3,4]$ & $\begin{array}{l}\text { Осуществление прямого иностранного инвестирования может ускорять экономический } \\
\text { прогресс в развивающихся странах через развитие собственных отраслей потребитель- } \\
\text { ских товаров с помощью сокращения времени, необходимого для наращивания конку- } \\
\text { рентоспособности в условиях рынка. }\end{array}$ \\
\hline С. Хаймер [13] & $\begin{array}{l}\text { Инвестор - главный игрок на рынке страны-реципиента, обладающий преимуществами } \\
\text { перед национальными производителями, использование которых может привести к вы- } \\
\text { теснению последних. }\end{array}$ \\
\hline Р. Кейвз $[8,9]$ & $\begin{array}{l}\text { Прямой эффект от ПИИ выражается в пополнении государственного бюджета за счет } \\
\text { дополнительных налоговых выплат, а также в создании новых рабочих мест, что в ре- } \\
\text { зультате снижает уровень безработицы. Косвенный эффект предполагает усиление кон- } \\
\text { куренции на рынке сбыта и ориентирование местных производителей на технологиче- } \\
\text { скую составляющую. }\end{array}$ \\
\hline $\begin{array}{l}\text { Haddad и Harrison, } \\
\text { Aitken и Harrison }[7,11]\end{array}$ & $\begin{array}{l}\text { Существует отрицательная корреляция между производительностью отечественных } \\
\text { предприятий и присутствием иностранных компаний на местном рынке. }\end{array}$ \\
\hline A. Kokko [14] & $\begin{array}{l}\text { Положительный косвенный эффект от ПИИ для страны-реципиента будет возможен } \\
\text { только в том случае, если в данном сегменте она уже обладает достаточно сильным уров- } \\
\text { нем технологического развития, а уровень конкуренции способен сдержать иностранного } \\
\text { инвестора от завоевания всего рынка. В остальных случаях нельзя говорить о «переливе» } \\
\text { технологии. }\end{array}$ \\
\hline Р. Пребиш [5] & $\begin{array}{l}\text { Прямое иностранное инвестирование является особым способом эксплуатации } \\
\text { «периферии» «центром» за счет «выкачивания» доходов. }\end{array}$ \\
\hline
\end{tabular}

Обобщая вышесказанное, можно выделить следующую структуру эффектов ПИИ на экономику страны-реципиента:

Положительное воздействие может заключаться в следующих аспектах: повышении количества рабочих мест и, как следствие, снижении уровня безработицы; развитии инфраструктуры; технологическом обмене и росте числа высококвалифицированной рабочей силы; повышении уровня заработной платы в определенном секторе экономики; развитии смежных отраслей; увеличении налоговых поступлений в бюджет государства.

Результатами отрицательного воздействия ПИИ могут оказаться: монополизация внутреннего рынка; отрицательное сальдо платежного баланса; ухудшение экологии [6]; потеря экономической и политической независимости; вытеснение местных производителей; изменение структуры экономики в целом.

2. Оценка эффектов, связанных с уровнем конкуренции национальных и иностранных инвестиций на внутреннем рынке России.

Гипотезу, поставленную в рамках решения данной задачи можно сформулировать следующим образом:

НО: Поток ПИИ в экономику принимающей страны способствует более активному вовлечению национальных инвестиций в производственный процесс. Имеет место «положительный эффект вовлечения».

H1: Поток ПИИ в экономику принимающей страны способствует нарастанию конкурирования внутренних и внешних инвестиций, иностранные инвестиции вытесняют внутренние. Имеет место «отрицательный эффект вытеснения».

Проверка гипотезы и количественная оценка этих эффектов заключается в определении и интерпретации коэффициентов абсолютной $(\mathrm{Et})$ и относительной $(\mathrm{Gt})$ эластичности национальных (внутренних) инвестиций по иностранным, подробно рассмотренным в работах [1]. Расчет абсолютной и относительной эластичности осуществляется по форм. 1, 2:

$$
\begin{aligned}
& \mathrm{E}_{\mathrm{t}}=\Delta \mathrm{L}_{\mathrm{t}}^{\mathrm{A}} / \Delta \mathrm{L}^{\mathrm{B}}, \\
& \mathrm{G}_{\mathrm{t}}=\left(\Delta \mathrm{L}_{\mathrm{t}}^{\mathrm{A}} / \mathrm{L}_{\mathrm{t}}^{\mathrm{A}}\right) /\left(\Delta \mathrm{L}^{\mathrm{B}}{ }_{\mathrm{t}} / \mathrm{L}^{\mathrm{B}}{ }_{\mathrm{t}}\right),
\end{aligned}
$$

где $\Delta \mathrm{L}^{\mathrm{A}}$ - изменение доли годовых национальных инвестиций в величине ВВП страныреципиента ПИИ; 
$\Delta \mathrm{L}_{\mathrm{t}}^{\mathrm{B}}$ - изменение доли годовых иностранных инвестиций в величине ВВП страныреципиента ПИИ.

В рамках данной работы была выдвинута гипотеза о том, что наличие конкретных эффектов зависит от стадии жизненного цикла ПИИ на территории страны-реципиента. В связи с этим авторами выделяются основные стадии и критерии их идентификации [2].

Так, для стадии допуска инвестора характерна невыраженная тенденция роста системы в целом с выраженной разнонаправленной динамикой в отдельных точках роста системы (регионах). Это связано с тем, что на этой стадии происходит отбор «пилотных» регионов для инвестирования. На стадии активного размещения характер динамики элементов приближается к общим тенденциям изменений системы и имеет ярко выраженный характер активного роста, сопровождающийся повышательным трендом. Это связано с распространением импульса от точек роста на остальные элементы системы. Стадия функционирования отличается от стадии активного размещения затухающей тенденцией роста ПИИ в целом по системе, а также более низкой волатильностью динамики ПИИ в регионах, чем на стадии допуска. На этой стадии, как правило, рост ПИИ происходит за счет реинвестированных доходов в регионы, подтвердившие ожидания инвестора.

Учитывая вышесказанное, для того чтобы избежать эффекта искажающего усреднения и получить более достоверные результаты, рассчитаем приведенные выше показатели для двух периодов, характеризующихся различными трендами в динамике потоков прямых иностранных инвестиций: с 2004 по 2008 год и с 2009 по 2016 год. На рис. 1. можно заметить, что первый период с 2004 по 2008 год отличается значительным ростом прямых иностранных инвестиций (темп прироста за 4 года составил 396,3 \%), потоки прямых иностранных инвестиций имеют в рамках данного периода ярко выраженную тенденцию к росту. Второй период с 2009 по 2016 год характеризуется неустойчивой динамикой ПИИ (размах составляет 350,3 \% от минимального значения в 2015 году).

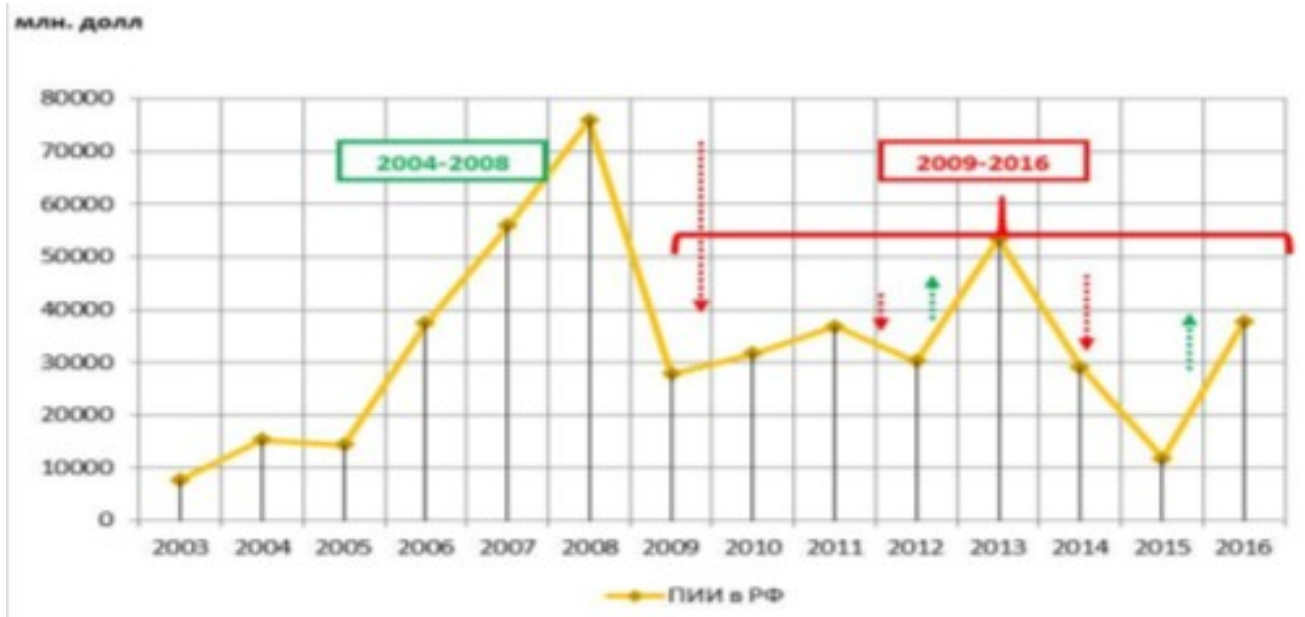

Pис. 1. Динамика сальдо ПИИ РФ с 2003 по 2016 год.

Источник: составлено авторами работы на основе статистических данных UNCTAD. Режим доступа: http:// unctadstat.unctad.org/EN/Index.html.

Таблий 2

Динамика удельного веса национальных и иностранных инвестиций в ВВП РФ

\begin{tabular}{|c|c|c|}
\hline Период & $L^{A}(\%)$ & $L^{\mathrm{B}}(\%)$ \\
\hline 2004 год & 16,03 & 1,59 \\
\hline 2008 год & 21,26 & 1,63 \\
\hline$\Delta$ (2004-2008 годы) & $+5,23$ & $+0,04$ \\
\hline 2009 год & 20,53 & 1,30 \\
\hline 2016 год & 17,58 & 11,06 \\
\hline$\Delta$ (2009-2016 годы) & $-2,95$ & $+9,76$ \\
\hline
\end{tabular}


Как видно из полученных данных, в период с 2004 по 2008 год абсолютная эластичность (Et) составила $130,75(5,23 / 0,04)$, а относительная $(\mathrm{Gt})-13,04((5,23 / 16,03) /(0,04 / 1,59))$. Так как полученные значения абсолютной и относительной эластичности выше нуля, то можно сделать вывод о том, что в экономике России в период с 2004 по 2008 год реализуется эффект мультипликации иностранных и внутренних инвестиций. Другими словами, ПИИ оказывают стимулирующий эффект на иностранные инвестиции, способствуя их более активному вовлечению в производственный процесс. Данную ситуацию можно охарактеризовать как наиболее комфортную для страны-реципиента, так как реализуется экономически эффективный баланс между иностранным и национальным воздействием на экономику России.

Данные, используемые для подсчета коэффициентов абсолютной и относительной эластичности, позволяют определить еще один важный показатель - объем инвестиций (капиталовложений) $\mathrm{mt}$, который содействовал бы экономическому росту «принимающей» экономики. Он рассчитывается по следующей формуле: $\mathrm{mt}=(1-\mathrm{LAt}) / \mathrm{LBt}$. Значение этого показателя выступает своеобразной «границей безубыточности» ПИИ, определяющей предельно допустимый, с точки зрения их максимальной эффективности, относительный объем. Пока этот объем для страны-реципиента ниже такого критического значения, можно рассчитывать на достаточно высокую отдачу от ПИИ. При превышении данного критического значения рост объема ПИИ будет экономически неэффективным. «Граница безубыточности» в 2004 году составила 52,8 ((1-0,1603)/0,0159), а в $2008-48,3((1-0,2126) / 0,0163)$. Это говорит о том, что максимальная эффективность может быть достигнута при увеличении инвестиций в 52,8 и 48,3 раза соответственно.

Абсолютная эластичность национальных инвестиций по иностранным в период с 2009 по 2016 год составила $-0,3(-2,95 / 9,76)$, а относительная - $(-0,02)((-2,95 / 20,53) /(9,76 / 1,3)$. Полученные результаты говорят о том, что за второй исследуемый период присутствовал «эффект вытеснения», а значит, реализуется ситуация, при которой национальные и иностранные инвестиции конкурируют между собой. Так как $\Delta$ LA2009-2016 < 0 (-2,95), а $\Delta$ LB2009-2016 $>0$ $(9,76)$, в экономике происходит процесс «выталкивания» внутренних инвестиций иностранными. Можно сделать вывод о том, что с 2009 по 2016 год ПИИ оказывают в целом депрессивное воздействие на инвестиционную деятельность в Российской Федерации и поэтому их увеличение крайне нежелательно. «Граница безубыточности» на 2009 год составляла 61,1 ((1$0,2053) / 0,0130)$, а на 2016 год - 7,45 ((1-0,1758)/0,1106). Превышение данного показателя в 2009 году по сравнению с 2016-м говорит о снижении отдачи от прямых иностранных инвестиций в Российской Федерации по отношению к предыдущим временным интервалам за счет «вытеснения» национальных инвестиций. Данная тенденция стала результатом одновременного снижения удельного веса национальных инвестиций и значительного увеличения удельного веса иностранных по отношению к ВВП страны-реципиента.

3. Оценка влияния ПИИ на экономику России на основе модифицированной разностной модели мультипликатора-акселератора.

Эта модель предполагает использование заложенных в объединенной модели экономического роста Харрода-Домара понятий мультипликатора $\left(\mathrm{m}_{\mathrm{t})}\right.$ и акселератора инвестиций $(\mathrm{Vt})[1$, $10]$.

Ключевым показателем данной модели является разностный диапазон приростной капиталоотдачи инвестиций национальных $\left(\beta \mathrm{t}^{\mathrm{A}}\right)$ и иностранных инвестиций $\left(\beta \mathrm{t}^{\mathrm{B}}\right)$, выражающий количество нового продукта, созданного единицей инвестиций:

$$
\begin{aligned}
& -\infty>\beta \mathrm{t}^{\mathrm{B}}-\beta \mathrm{t}^{\mathrm{A}}>\infty \\
& \beta \mathrm{t}^{\mathrm{B}}=\Delta \mathrm{Y}_{\mathrm{t}}^{\mathrm{B}} / \mathrm{J}_{\mathrm{t}}^{\mathrm{B}} \\
& \beta \mathrm{t}^{\mathrm{A}}=\Delta \mathrm{Y}_{\mathrm{t}}^{\mathrm{A}} / \mathrm{J}_{\mathrm{t}}^{\mathrm{A}} \\
& \mathrm{J}_{\mathrm{t}}=\mathrm{J}_{\mathrm{t}}^{\mathrm{B}}+\mathrm{J}_{\mathrm{t}}^{\mathrm{A}}
\end{aligned}
$$

где $\Delta \mathrm{Y}_{\mathrm{t}}^{\mathrm{A}}$ и $\Delta \mathrm{Y}_{\mathrm{t}}^{\mathrm{B}}$ - количество нового продукта (прирост объема ВВП), создаваемого в национальном и «зарубежном» секторах экономики страны-реципиента;

$\mathrm{J}_{\mathrm{t}}, \mathrm{J}_{\mathrm{t}}^{\mathrm{A}}$ и $\mathrm{J}_{\mathrm{t}}^{\mathrm{B}}$ - соответственно общие, национальные и иностранные инвестиции в странереципиенте.

Дальнейшая логика проверки гипотез относительно влияния ПИИ на экономику принимающей страны на основании системного анализа следующих дополнительных показателей мо- 
дели:

- темпа прироста нового продукта (ВВП) $(\lambda \mathrm{t})$

$$
\lambda \mathrm{t}=\Delta \mathrm{Y}_{\mathrm{t}} / \mathrm{Y}_{\mathrm{t}}
$$

- доли иностранных инвестиций сектора в общем объеме инвестиций в экономике страныреципиента $\left(\mathrm{n}_{\mathrm{t}}\right)$

$$
\mathrm{n}_{\mathrm{t}}=\mathrm{J}_{\mathrm{t}}^{\mathrm{B}} / \mathrm{J}_{\mathrm{t}}
$$

В результате проверяются 4 гипотезы:

Н0: начиональная экономика находится в состоянии рецессии, которая может быть преодолена в том числе за счет привлечения прямых иностранных капиталовложений $(\lambda t<0$; $n t=0 ; \beta t A<0)$.

H1: сектор с иностранным капиталом менее эффективен, чем сектор с начиональным капиталом в связи с тем, что приростная капиталоотдача иностранных инвестиций меньше, чем национальныхх. $(\lambda t>0 ; n t \neq 0 ; \beta t A>\beta t B>0)$.

H2: эффективность национального и «зарубежного» секторов экономики страныреципиента одинакова $(\lambda t>0 ; n t \neq 0 ; \beta t A=\beta t B>0)$.

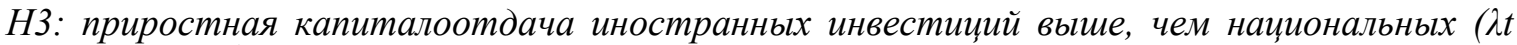
$\left.>0 ; n t \neq 0 ; \beta t^{B}>\beta t^{A}>0\right)$.

Мультипликатором-акселератором в данной модели выступает темп прироста производства, обусловленный изменением доли инвестиций «зарубежного» сектора страны-реципиента $\mathrm{Wt}$ :

где $\Delta \mathrm{n}_{\mathrm{t}}=\Delta \mathrm{J}_{\mathrm{t}}^{\mathrm{B}} / \mathrm{J}_{\mathrm{t}}$

$$
\mathrm{Wt}=\left[\left(\beta \mathrm{t}^{\mathrm{B}}-\beta \mathrm{t}^{\mathrm{A}}\right) \times \mathrm{St} \times \Delta \mathrm{n}_{\mathrm{t}}\right] /(1+\lambda \mathrm{t})
$$

При $\mathrm{Wt}>0$ существующая тенденция способствует повышению темпов эконмического роста (при $\lambda \mathrm{t}>0$ ) или смягчает кризисные тенденции (при $\lambda \mathrm{t}<0$ ). Соответственно, при $\mathrm{Wt}<0$ замедляется экономический рост (при $\lambda \mathrm{t}>0$ ) или усугубляется производственная рецессия (при $\lambda \mathrm{t}<0)$.

В целом по России за период с 1999 по 2015 год расчеты с учетом выделенных инвестиционных циклов приведены в табл. 3 .

\section{Результаты расчетов коэффициентов модели Харрода-Домара}

\begin{tabular}{|c|c|c|c|}
\hline Параметры модели & $1999-2003$ & $2004-2008$ & $2009-2015$ \\
\hline$\beta \mathrm{t}(\mathrm{A})$ & 7,68 & 7,04 & 3,42 \\
\hline$\beta \mathrm{t}(\mathrm{B})$ & 21,66 & 54,15 & 11,50 \\
\hline $\mathrm{J}(\mathrm{t})$ & 736999 & 2394338 & 8435589 \\
\hline $\mathrm{Y}(\mathrm{t})$ & 4149289 & 10742423 & 32072552 \\
\hline $\mathrm{n}(\mathrm{t})$ & 0,09 & 0,09 & 0,06 \\
\hline $\mathrm{S}(\mathrm{t})$ & 0,18 & 0,22 & 0,26 \\
\hline $\mathrm{B}(\mathrm{t})$ & 8,95 & 11,13 & 3,90 \\
\hline$\lambda(\mathrm{t})$ & 1,59 & 2,48 & 1,03 \\
\hline $\mathrm{W}(\mathrm{t})$ & 0,84 & $-0,05$ & $-0,01$ \\
\hline
\end{tabular}

Как видно из результатов расчетов, приростная капиталоотдача иностранных инвестиций всегда была выше национальных в несколько раз, однако к 2015 году она несколько снизилась и приблизилась к капиталоотдаче национальных инвестиций. Иностранные инвестиции способствовали экономическому росту России в целом лишь в период с 1999 по 2003 год (стадия проникновения). Однако, учитывая сильную степень дифференциации распределения иностранных инвестиций в регионы РФ, признавать безусловными полученные выводы представляется спорным, поскольку в этом случае мы можем столкнуться с «проклятием» средней, то есть со случаем, когда тенденция крупнейшего региона-донора искажает общий вывод. Поэтому дальнейшие расчеты были проведены по основным регионам-реципиентам (доля потоков иностранных 
инвестиций, в которых находилась на уровне более 5 \%). В результате было выделено несколько групп, имеющих различные тенденции:

- регионы, в которых наблюдается замедление экономического роста как в период с 2004 по 2008 год, так и с 2009 по 2015 год - Московская область, Сахалинская область, Тюменская область;

- регионы, в которых наблюдается ускорение экономического роста на протяжении всего исследуемого периода: Санкт-Петербург, Ленинградская область, Челябинская, Свердловская области и прочие регионы с низкой долей инвестиционных поступлений в общем объеме по России;

- регионы, в которых наблюдается положительная тенденция, сопровождающаяся сначала замедлением, а потом ускорением экономического роста - Красноярский край, Липецкая область;

- отрицательная тенденция наблюдается только в одном регионе - г. Москве. Именно этот регион и определяет тенденцию по всей России за счет весомого удельного веса в общем объеме иностранных инвестиций.

Заключение. По результатам проведенного исследования мы пришли к выводу о том, что к основным причинам возникновения столь полярных суждений об эффектах прямого иностранного инвестирования на экономику страны-реципиента относятся:

1. Различия в выборе базовых теорий для оценки эффектов.

2. Различия в исходных данных (например, из-за различного качества данных или различий в составе выборки, такие как различные наборы стран).

3. Различия в характере анализируемых трендов ПИИ.

Полученные результаты расчетов, проведенных на основе данных по РФ, показали, что эффекты от ПИИ зависят от стадии жизненного цикла.

В рамках стадии активного привлечения иностранных инвесторов ПИИ оказывают стимулирующий эффект на национальные иностранные инвестиции, способствуя их более активному вовлечению в производственный процесс, однако на второй стадии - стадии размещения наблюдается «эффект вытеснения» национальных инвестиций иностранными, а значит, реализуется сценарий, при котором национальные и иностранные инвестиции конкурируют между собой.

На протяжении всего анализируемого периода приростная капиталоотдача иностранных инвестиций всегда была выше национальных в несколько раз, что свидетельствует о более совершенных управленческих процессах в данных кампаниях и о возможном потенциале в отношении стимулирования экономического роста в РФ. Однако расчеты, выполненные в целом по РФ, показали, что иностранные инвестиции способствовали экономическому росту в России в целом лишь в период с 1999 по 2003 год. Выявленное противоречие объясняется сложившейся дифференциацией потоков и различным влиянием прямых иностранных инвестиций на экономический рост различных регионов РФ. Общая тенденция обусловлена тенденцией всего одного региона - г. Москвы, за счет весомого удельного веса в общем объеме иностранных инвестиций (в среднем около 40 \% на протяжении анализируемого периода), в то время как в других регионах по-прежнему наблюдается положительный эффект прямых иностранных инвестиций на экономический рост, отличающийся лишь разной степенью интенсивности. В сложившейся ситуации, на наш взгляд, более эффективной становится модель адресной региональной государственной политики стимулирования иностранных инвестиций, которая позволит максимально использовать положительный эффект в конкретных регионах РФ с учетом стадии жизненного цикла иностранных инвестиций в рамках данной территории и нивелировать отрицательные эффекты, наблюдающиеся в других регионах.

Исследование выполнено при финансовой поддержке РФФИ в рамках проекта проведения научных исследований «Исследование потенциала отечественных производителей по обеспечению импортозамещения на потребительском рынке с использованием технологий Big Data», проект № 17-02-00718.

Исследование выполнено при финансовой поддержке РФФИ в рамках проекта проведения научных исследований «Разработка подходов к созданию системы оценки состояния и определения перспективных направлений развития научной сферы», проект № 16-02-00407. 
Лuтература

1. Иванов В. А., Головастова Т. И., Дыбов А. М. Иностранные инвестиции. - Институт экономики и управления ГОУ ВПО «УдГУ», 2009. - 159 с.

2. Матраева Л. В. Методология статистического анализа инвестиционной привлекательности регионов РФ для иностранных инвесторов. М.: Дашков и К, 2013. - 312 c.

3. Пестова А. А., Сухарева И. О., Солнцев О. Г. О стимулировании притока прямых иностранных инвестиций в российскую экономику с иелью повышения «качества» экономического роста // Проблемь прогнозирования. - 2011. - № 1. - С. 136-154.

4. Пилипенко И. В. Конкурентоспособность стран и регионов в мировом хозяйстве: теория, опыт мальхх стран Западной и Северной Европьл. - Смоленск: Ойкумена, 2005. - 496 c.

5. Пребиш Р. Периферийный капитализм: есть ли ему альтернатива? - М.: ИЛА, 1992. - 337 с. (Capitalismo periferico. Crisis y transformacion, 1981).

6. Рогатнев Н. С. Эффекты прямых иностранных инвестищий в развивающихся странах: типология, моделирование, регулирование/ дисс. на соискание ученой степени к. э. н.: 08.00.01. - Воронеж, 2015. $150 \mathrm{c}$.

7. Aitken B. J. and Harrison A. E. (1999). Do domestic firms benefit from direct foreign investment? Evidence from Venezuela. American Economic Review. No. 89. P.605-618.

8. Caves R. E. (1974). "Multinational Firms, Competition, and Productivity in Host Country Markets», Economica. Vol. 41. P. 176-93, 205.

9. Caves R. E. (1993). "Japanese Investments in the US: Lessons for the Economic Analysis of Foreign Investment». The World Economy 16. P. 279300.

10. Ekanayake E. M., Chatrna D. (2010) The effect of foreign aid on economic growth in developing countries // Journal of International Business and Cultural Studies. [An electronic resource]. Access mode: https:// www.researchgate.net/

publication/255605340_The_effect_of_foreign_aid_on_economic_growth_in_developing_countries, free. Heading from the screen.

11. Haddad M. and Harrison A. (1992). "Are Positive Spillovers from Direct Foreign Investment? Evidence from Panel Data for Morocco»// Journal of Development Economics, 42:51-74.

12. Heng T. M. Learning curves and productivity in Singapore manufacturing industries // Second Annual Conference of the Academic Network for Development in Asia (ANDA). 2010.

13. Hymer S.H. (1972) "The Multinational Corporation and the Law of Uneven Development» in J. N. Bhagwati (Ed.), Economics and World Order, New York, NY: MacMillan. P. 113-40.

14. Kokko A. (1994). Technology, market characteristics, and spillovers. Journal of Development Economics, 43, 279-293.

15. Lemi A. Foreign direct investment, host country productivity And export: the case of US and Japanese Multinational affiliates // Journal of economic development. 2004. T. 29. No. 1. P. 163-187.

16. Meysam Amani (2014) Critical Study of Foreign Direct Investment on Economic Development. Scholars' Press. 224 p.

17. Vernon R. International Investment And International Trade In The Product Cycle // Quarterly Journal Of Economics. V. 80 (2). 1966. P. 190-207.

18. Vintila D. (2010) Foreign Direct Investment Theories: An Overview of the Main FDI Theories // European Journal of Interdisciplinary Studies. Vol. 2 Iss.2. P. 104-110.

19. [An electronic resource]. Access mode: http://www.investmentsandincome.com/investments/oliparadigm.html, free. Heading from the screen.

20. Zorska A. (2005) Foreign Direct Investment and Transformation: Evolution and Impacts in the Polish Economy. Eastern European Economics. Vol. 43. No. 2.

\section{References:}

1. [An electronic resource]. Access mode: http://www.investmentsandincome.com/investments/oliparadigm.html, free. Heading from the screen.

2. Aitken B. J. and Harrison A. E. (1999). Do domestic firms benefit from direct foreign investment? Evidence from Venezuela. American Economic Review. No. 89. P. 605-618.

3. Caves R. E. (1974). "Multinational Firms, Competition, and Productivity in Host Country Markets». Economica. Vol. 41. P. 176-93, 205.

4. Caves R. E. (1993). "Japanese Investments in the US: Lessons for the Economic Analysis of Foreign Investment». The World Economy 16. P. 279300

5. Ekanayake E. M., Chatrna D. (2010) The effect of foreign aid on economic growth in developing countries // Journal of International Business and Cultural Studies. [An electronic resource]. Access mode: https:// www.researchgate.net/

publication/255605340_The_effect_of foreign_aid_on_economic_growth_in_developing_countries, free. Heading from the screen.

6. Haddad M. and Harrison A. (1992). "Are Positive Spillovers from Direct Foreign Investment? Evidence from Panel Data for Morocco». Journal of Development Economics, 42:51-74.

7. Heng T. M. Learning curves and productivity in Singapore manufacturing industries // Second Annual Conference of the Academic Network for Development in Asia (ANDA). 2010.

8. Hymer S. H. (1972). "The Multinational Corporation and the Law of Uneven Development» in J. N. Bhagwati (Ed.). Economics and World Order, New York, NY: MacMillan. P. 113-40.

9. Ivanov V. A., Golovastova T. I., Dybov A. M. Foreign investments. Institute of Economics and Management, 


\section{МАТРАЕВА Л.В., АЛЯББЕВА А.В. \\ МАКРОЭКОНОМИЧЕСКИЕ ЭФФЕКТЫ ПРЯМОГО ИНОСТРАННОГО ИНВЕСТИРОВАНИЯ В РЕГИОНЫ \\ РОССИЙСКОЙ ФЕДЕРАЦИИ}

GOU VPO «UdSU», 2009. $159 \mathrm{p}$.

10. Kokko A. (1994). Technology, market characteristics, and spillovers. Journal of Development Economics. 43. P. 279-293.

11. Lemi A. Foreign direct investment, host country productivity And export: the case of US and Japanese Multinational affiliates // Journal of economic development. 2004. T. 29. No 1. P. 163-187.

12. Matraeva L. V. Methodology of statistical analysis of the investment attractiveness of the regions of the Russian Federation for foreign investors. Moscow: Dashkov and K, 2013. 312 p.

13. Meysam Amani. (2014). Critical Study of Foreign Direct Investment on Economic Development. Scholars' Press. 224 p.

14. Pestova A. A., Sukhareva I. O., Solntsev O. G. On stimulating the influx of foreign direct investment in the Russian economy with a view to raising the «quality» of economic growth // Problems of forecasting. 2011. No. 1. P. 136-154.

15. Pilipenko I. V. Competitiveness of countries and regions in the world economy: theory, experience of small countries of Western and Northern Europe. Smolensk: Oykumena, 2005. 496 p.

16. Prebis R. Peripheral capitalism: is there an alternative to it? Moscow: ILA, 1992. 337 p (Capitalismo periferico, Crisis y transformacion, 1981).

17. Rogatnev N. S. Effects of foreign direct investment in developing countries: typology, modeling, regulation / diss. for the academic degree of the candidate of economic sciences: 08.00.01. Voronezh, 2015. $150 \mathrm{p}$.

18. Vernon R. International Investment And International Trade In The Product Cycle // Quarterly Journal Of Economics. Vol. 80 (2). 1966. P. 190-207.

19. Vintila D. (2010) Foreign Direct Investment Theories: An Overview of the Main FDI Theories // European Journal of Interdisciplinary Studies. Vol. 2 Iss. 2. P. 104-110.

20. Zorska A. (2005) Foreign Direct Investment and Transformation: Evolution and Impacts in the Polish Economy. Eastern European Economics. Vol. 43. No. 2. 